\title{
Z-ADALINE based high-precision wide-frequency signal measurement algorithm for power electronic power grid
}

\author{
Wenxiu Wang ${ }^{1,2}$, Shuguo Pan ${ }^{2, *}$, Jiawei Peng ${ }^{2}$, Jian Shen $^{3,4}$, Min Zhang ${ }^{3,4}$, Wang Gao ${ }^{2}$, Chenglin Xia ${ }^{3,4}$ \\ ${ }^{1}$ Jinling Institute of Technology, Nanjing, China \\ ${ }^{2}$ Southeast University, Nanjing, China \\ ${ }^{3}$ Nari Technology CO., Ltd, Nanjing China \\ ${ }^{4}$ State Key Laboratory of Smart Grid Protection and Control, Nanjing, China
}

\begin{abstract}
Intermittent wind power, photovoltaic and other renewable energies have been paralleled, which makes the phenomena of high-order harmonics and simple harmonics more and more serious in the power system, showing a wide-frequency trend. The existing measurement algorithms mainly aim at signals in midfrequency and low-frequency. Besides, they are lack of a uniform high-precision algorithm for widefrequency measurement. To solve this problem, we propose a high-precision algorithm based on Z-ADALINE. Firstly, Zoom FFT algorithm is used to analyze original sampled signals. This step enables the refinement of its frequency spectrum, and obtains accurate frequency measurement results. At this time, the number of frequencies can also be determined. Secondly, the result of Zoom FFT is used as the input of the adaptive linear neural network(ADALINE). ADALINE can estimate amplitude and phase with high precision. The simulation results show that the proposed algorithm can realize high-precision measurement of frequency, amplitude and phase of wide-frequency signal effectively. Among them, the frequency resolution can be up to $0.3 \mathrm{~Hz}$. The amplitude error is within $1 \mathrm{~V}$. Phase error is less than $0.6^{\circ}$. The results may provide some significant references for practical wide-frequency signal measurement in power electronic power grid.
\end{abstract}

\section{Introduction}

Harmonics and inter-harmonics in power system have always been an attractive topic ${ }^{[1-2]}$. Harmonics increase circuit loss, affect circuit performance and the normal usage of computers and other equipment, which would shorten the life of the equipment in the long run ${ }^{[3]}$. Interharmonics cause voltage fluctuation, sub-synchronous oscillation, zero-cross bias, communication interference and power grid breakdown ${ }^{[4]}$. In recent years, intermittent wind power, photovoltaic and other renewable energies have been paralleled, injecting large amounts of low and high frequency components into the power grid. Interharmonics and high harmonics broaden the research scope of power frequency frequency ${ }^{[5-6]}$, showing a widefrequency trend ${ }^{[7]}$. Existing frequency estimation algorithms cannot solve the problem of wide-frequency signal estimation. High-precision algorithms for widefrequency signal measurement are urgently needed ${ }^{[8-9]}$. Additionally, parameters including the center frequency, the frequency bandwidth, and magnification of Zoom FFT are set manually. It is difficult to meet the requirements of adaptive ability and limits the application of Zoom FFT.

Fast Fourier Transform $(\mathrm{FFT})^{[10]}$ and improved algorithms are widely used among traditional power signal estimation. These algorithms have advantages in computation and hardware implementation. However, when input frequencies are not an integral multiple of the FFT resolution, the energy of the signal would range in entire frequency domain. In this case, the frequency points with relatively small amplitudes will be submerged and cannot be observed. This will cause spectrum leakage. Multiple windowing algorithms can effectively alleviate this problem, but with the sampling frequency increases, the resolution decreases ${ }^{[11]}$. When measuring the widefrequency signal with the same sampling length, the increase in sampling frequency decreases the frequency resolution of FFT, resulting in spectral line concentration or indistinguishable spectrum. However, Zoom FFT can effectively improve the frequency resolution of widefrequency signal estimation ${ }^{[12]}$, but it still remains problems such as spectral leakage, and cannot accurately estimate the amplitude and phase.

In the field of deep learning, ADALINE neural network has emerged along with the development of artificial intelligence ${ }^{[13]}$. It has been widely used in adaptive processing of signal. However, ADALINE cannot realize frequency adjustment ${ }^{[14]}$. It's easily to produce large error when the number and frequencies estimate are inaccurate. To solve this problem, Goh et al. ${ }^{[15]}$ proposed a frequency estimation algorithm based on FFT-ADALINE. They use FFT to obtain the number of frequencies, and in combination with ADALINE to

*Corresponding author's e-mail:psg@seu.edu.cn 
improve the accuracy of frequency, amplitude, and phase estimation. In order to further reduce this error, Chen et al. ${ }^{[16]}$ propose improved enhanced ADALINE neural network. They used Root-MUSIC based on characteristic subspace to measure the initial frequencies of the inter-harmonics. They improved the structure of ADALINE neural network structure in order to make it capable of adjusting frequency. However, the convergence and antiinterference performances still need to be improved. As for the deficiency of MUSIC, the researchers propose an ESPRIT-ADALINE algorithm [17], whose estimation accuracy is better than that of MUSIC. However, when estimating wide-frequency signal, the ESPRIT-based spectral estimation algorithms are difficult to maintain a fixed sampling frequency. Therefore, they are difficult to realize high-precision estimation, While Zoom FFT can be used for applications with fixed sampling frequency.

From the above reasons, this paper proposes an estimation algorithm based on Zoom FFT and ADALINE. The adaptive ability of Zoom FFT is designed to realize accurate frequency estimation. Z-ADALINE realizes the high-precision estimation of wide-frequency signal's frequency, amplitude, and phase. It meets the practical requirements of fixed sampling frequency of equipment.

\section{Z-ADALINE based high-precision wide-frequency signal estimation algorithm}

The specific frequency range of wide-frequency signal has not been defined certainly. In this paper, the frequency range is mainly based on the $50 \mathrm{~Hz}$ power frequency, including the frequency of low frequency oscillation, subsynchronous oscillation and super synchronous oscillation. High frequency signals beyond the 50th harmonic are temporarily excluded. Therefore, it is determined that the frequency range that this paper focuses on is from 0 to $2.5 \mathrm{kHz}$. Based on this premise, we explore high-precision estimation algorithm for the frequency, amplitude, and phase of signals in this range.

\subsection{Multiple modulation zoom spectrum analysis algorithm (Zoom FFT)}

When it comes to Zoom FFT, we firstly use FFT to roughly estimate the signal frequency spectrum. Considering the influence of noise and all error factors, a frequency range including the frequency that we measure is estimated. The implementation steps of Zoom FFT include complex modulation frequency shift, low-pass filter, resampling, FFT spectrum analysis and frequency adjustment ${ }^{[18]}$. The detailed contents are as follows:

Complex modulation frequency shift. Assuming that the power signal is $x_{0}(t)$, sampling frequency is $f_{s}$, sampling sequence obtained after sampling is $x_{0}(n)$, $n=0,1, \ldots, N-1$, where, $\mathrm{N}$ is the number of signal points that FFT analysis. Zoom frequency range is $f_{1} \sim f_{2}$. Center frequency of the range can be defined as $f_{c}=\left(f_{1}+f_{2}\right) / 2$ and magnification is $\mathrm{D}$, Then, after doubling the zoom frequency range, this paper gets $f_{2}{ }^{\prime}-f_{1}{ }^{\prime}=2\left(f_{2}-f_{1}\right)$. Then $\mathrm{D}$ can be Expressed as $D=f_{s} / f_{2}{ }^{\prime}-f_{1}{ }^{\prime}=f_{s} / 2\left(f_{2}-f_{1}\right)$.

After complex modulating, the center frequency of zoom frequency range is moved to zero point ${ }^{[19]}$ and get $x(n)$ :

$$
\begin{aligned}
x(n)= & x_{0}(n) e^{-j 2 \pi n f_{c} / f_{s}} \\
= & x_{0}(n) \cos \left(2 \pi f_{c} / f_{s}\right)-j x_{0}(n) \sin \left(2 \pi f_{c} / f_{s}\right) \\
= & x_{0}(n) \cos \left(2 \pi n L_{0} / N\right)-j x_{0}(n) \sin \left(2 \pi n L_{0} / N\right) \\
& X(k)=X_{0}\left(k+L_{0}\right)
\end{aligned}
$$

Where $X(k)$ is the discrete spectrum of $x(n), X_{0}(k)$ is the discrete spectrum of $x_{0}(n) \cdot f_{s}=N \Delta f$, where $\Delta f$ is frequency resolution. $L_{0}=f_{c} / \Delta f$ is the index number of spectral lines in original spectrum of $f_{c}$.

Low-pass filter. It is used to obtain the signal in zoom frequency range. This can effectively remove the frequency components outside the frequency band that the signal needs to refine. The frequency response of the filter is:

$$
\begin{aligned}
& Y_{L}(k)=X(k) H(k)=X_{0}\left(k+L_{0}\right) H(k), \\
& k=0,1, \ldots, \frac{N}{2}, \ldots, N-1
\end{aligned}
$$

After Low-pass filter, the signal sequence output is:

$$
y_{L}(n)=\frac{1}{N} \sum_{k=0}^{N-1} Y(k) W_{N}^{-n k}=\frac{1}{N} \sum_{k=0}^{N-1} X(k) H(n) W_{N}^{-n k}
$$

Resampling. After complex modulating and low-pass filter, the frequency range reduced by $1 / D$ times. The resampling frequency is $f_{s}{ }^{\prime}=f_{s} / D$.

$$
g(m)=y(D m)=\frac{1}{N}\left[\sum_{p=0}^{N / 2-1} X_{0}\left(p+L_{0}\right) W^{-p m}+\sum_{p=N / 2}^{N-1} X_{0}\left(p-N+L_{0}\right) W^{-p m}\right]
$$

FFT spectrum analysis. $g(m)$ is analyzed by FFT. The frequency resolution increases by $D$ times, that is $\Delta f^{\prime}=f_{s}{ }^{\prime} / N=f_{s} /(N D)=\Delta f / D$. The discrete spectrum of $g(m)$ is as follows:

$$
\begin{aligned}
G(k) & =\sum_{m=0}^{N-1} g(m) W_{N}^{m k} \\
& =\left\{\begin{array}{l}
\frac{1}{D} X_{0}\left(k+L_{0}\right), k=0,1, \ldots, \frac{N}{2}-1 \\
\frac{1}{D} X_{0}\left(k+L_{0}-N\right), k=\frac{N}{2}, \frac{N}{2}+1, \ldots, N-1
\end{array}\right.
\end{aligned}
$$

Frequency adjustment. This step reorders the last results. The result of resampling is a complex-valued sequence, and all data is useful information when performing FFT calculations. Because it uses the modulation frequency $f_{k}$ as the new zero frequency, 
there is actually asymmetry, so the general complex result at the negative frequency is valid. In the transformation result of the MATLAB function FFT, the data corresponding to the positive frequency is stored in the first half part of the result vector in the forward direction from the beginning, and the data of the complex frequency is stored in the second half part of the data in the reverse order from the tail. section. After moving the spectral line of $G(k)$ to the actual frequency, the zoom band is obtained as:

$$
X_{0}(k)=\left\{\begin{array}{l}
D G\left(k-L_{0}\right), k=L_{0}, L_{0}+1, \ldots, L_{0}+\frac{N}{2}-1 \\
D G\left(k-L_{0}+N\right), k=L_{0}-\frac{N}{2}, \ldots, L_{0}-1
\end{array}\right.
$$

Finally, high-precision frequency estimation results zooming by $\mathrm{D}$ times is obtained.

\subsection{Adaptive linear neuron network}

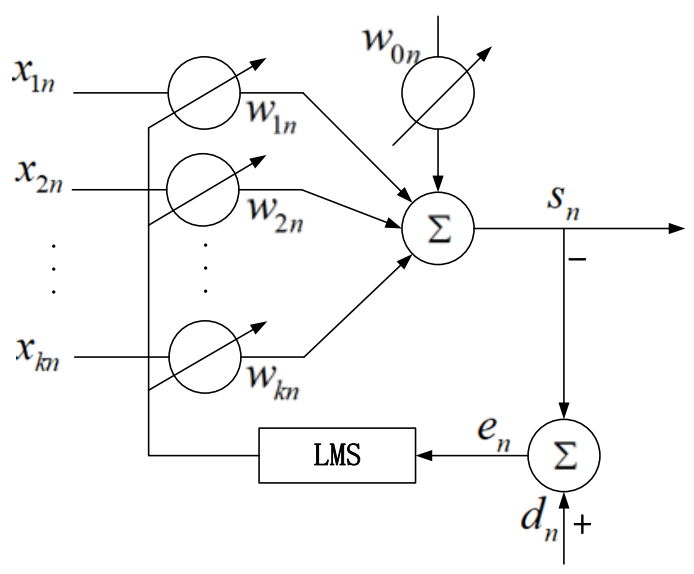

Fig. 1 ADALINE neural network structure

The basic structure of the ADALINE neural network can be shown as Fig. $1^{[20]}$. Where, $X_{n}=\left[x_{0 n}, x_{1 n}, x_{2 n}, \cdots, x_{k n}\right]^{T}$ is the input, also known as the mode vector. $W_{n}=\left[w_{0 n}, w_{1 n}, w_{2 n}, \cdots, w_{k n}\right]^{T}$ is the weight vector. $s_{n}$ is the output of the neural network. $d_{n}$ is the expected output, $e_{n}$ is the error function ${ }^{[21]}$. The output can be expressed by a linear combination of input and weight vector ${ }^{[22]}$.

$$
s_{n}=X_{n}^{T} W_{n}
$$

Using the error function $e_{n}$, the difference between the network output $s_{n}$ and the expected output $d_{n}$, the network's weight vector $W_{n}$ can be adjusted. The Least Mean Square (LMS) is the most common weight adjustment algorithm. After the iteration convergence of the weights, the network output $s_{n}$ gradually approximates the expectation.

The power signal can be expressed as:

$$
y(t)=d_{0}+\sum_{m=1}^{M} A_{m} \sin \left(2 \pi f_{m} t+\varphi_{m}\right)
$$

Where $d_{0}$ is the DC component, $A_{m}, f_{m}$ and $\varphi_{m}$ are the amplitude, frequency and initial phase of the $m$ th signal component.

The sampling sequence of $y(t)$ is ${ }^{[23]}$ :

$$
\begin{aligned}
y(n) & =\sum_{m=1}^{M} A_{m} \sin \left(2 \pi f_{m} n+\varphi_{m}\right)+v(n) \\
& =\sum_{m=1}^{M}\left[a_{m} \cos \left(\omega_{m} n\right)+b_{m} \sin \left(\omega_{m} n\right)\right]+v(n)
\end{aligned}
$$

Where $a_{m}=A_{m} \sin \varphi_{m}$ and $b_{m}=A_{m} \cos \varphi_{m}$. Therefore, when the algorithm is applied to power signal estimation, the input vector and weight vector of ADALINE are expressed as follows.

$$
\begin{gathered}
X_{n}=\left[x_{0 n}, x_{1 n}, x_{2 n}, \cdots, x_{k n}\right]^{T} \\
=\left[1, \cos \left(\omega_{1} n\right), \sin \left(\omega_{1} n\right), \cdots, \cos \left(\omega_{M} n\right), \sin \left(\omega_{M} n\right)\right]^{T} \\
W_{n}=\left[w_{0 n}, w_{1 n}, w_{2 n}, \cdots, w_{k n}\right]^{T} \\
=\left[d_{0}, a_{1}, b_{1}, \cdots, a_{M}, b_{M}\right]^{T}
\end{gathered}
$$

The actual sampling data $y(n)$ is used as the expected signal and the output of the network is $\hat{y(n)}$. In the process of network parameter modification, the output continuously approximates to the expectation. Iteration ends when the error is below the threshold or when the algorithm reaches the maximum number of iterations. Finally, the weight information $a_{m}, b_{m}(m=1,2, \cdots, M)$ are obtained. The amplitude and phase information of harmonics and inter-harmonics are obtained.

$$
\begin{gathered}
f_{m}=\omega_{m} / 2 \pi \\
A_{m}=\sqrt{a_{m}^{2}+b_{m}^{2}} \\
\varphi_{m}=\arctan \frac{a_{m}}{b_{m}}
\end{gathered}
$$

ADALINE is used for harmonic analysis without the sample training process. Namely, there isn't the test of data set by training set. In practice, it is only necessary to make constant error reverse transmission and weight adjustment.

\subsection{Z-ADALINE}

An algorithm for accurately estimating the frequency, amplitude, and phase with practical value is the focus of this paper. The conventional FFT+ADALINE algorithm is affected by the signal number and frequency accuracy, which are estimated by FFT. This algorithm is already difficult to meet the new demands of the wide-frequency signal measurement. The MUSIC+ADALINE and 
ESPRIT+ADALINE algorithms obtain good results in low and medium frequency, but it is difficult to maintain the $12.8 \mathrm{kHz}$ sampling frequency in the wide-frequency signal measurement. Combining the strengths of Zoom FFT and ADALINE, this paper carries out the study. However, the zoom frequency range and center frequency of Zoom FFT are set manually while the actual signal parameters are difficult to predict. This requires the adaptive ability of Zoom FFT ${ }^{[24]}$. Therefore, this paper proposes an adaptive Z-ADALINE to improve estimation accuracy.

This paper first designs Zoom FFT algorithm with adaptive ability. The function of Zoom-FFT is to locally amplifying the frequency of the signals. Therefore, the band of interest can obtain a higher frequency resolution. There are several algorithms to realize the FFT refinement function, such as frequency shift, phase compensation and maximum entropy spectrum algorithms. We choose the frequency shift algorithm. The specific implementation process is as follows.

1) The zoom frequency range and center frequency adaptive determination algorithm: The signal frequency shift involves two parts.

One is the frequency that the FFT can discriminate, which can be retained directly without zooming. This part has the distinctive feature of narrow bandwidth. At the same time, we follow the principles of sampling theorem. In order to prevent the frequency of the sampled signal from being confused, it needs to be filtered by a low-pass anti-aliasing filter or a sufficiently high sampling frequency $f_{s}$.

The other part requires complex modulation frequency shift. The zoom frequency range and center frequency need determining. Firstly, this paper analyses the data processed by FFT and obtains the frequency components $f^{\prime}=\left[f_{1}{ }^{\prime}, f_{2}{ }^{\prime}, \ldots, f_{n}{ }^{\prime}\right]$. The peak frequency is treated as the center frequency. Then, the algorithm judges whether the bandwidth exceeds the FFT resolution range, when the amplitude decreases to $1 / 10$ of the amplitude at the center frequency, that is the frequency range of $-20 \mathrm{~dB}$ such as $\left[f_{21}{ }^{\prime}, f_{22}{ }^{\prime}\right]$ corresponding to $f_{2}{ }^{\prime}$. If it goes far beyond the range, the zoom is required here and the center frequency is $f_{2}{ }^{\prime}$, zoom frequency range is $\left(f_{22}{ }^{\prime}-f_{21}{ }^{\prime}\right)$, as shown in Fig. 2.

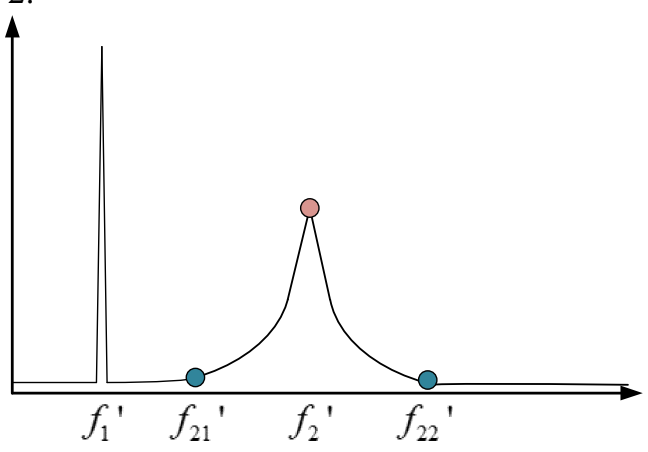

Fig. 2 Adaptive zoom frequency range analysis

2) The adaptive ability of magnification $D:$ After determining the center frequency and the range, the sampling frequency decreases by $D$ times, when resampling the signal after frequency shifting. Magnification should be no less than $\left(f_{22}{ }^{\prime}-f_{21}{ }^{\prime}\right) / f_{r}$, where $f_{r}$ is the desired frequency resolution. In real application, the specific value of $D$ can be adjusted according to the actual effect.

The algorithm in this paper as shown in Fig. 3 combines Zoom FFT possessing adaptive ability with ADALINE.

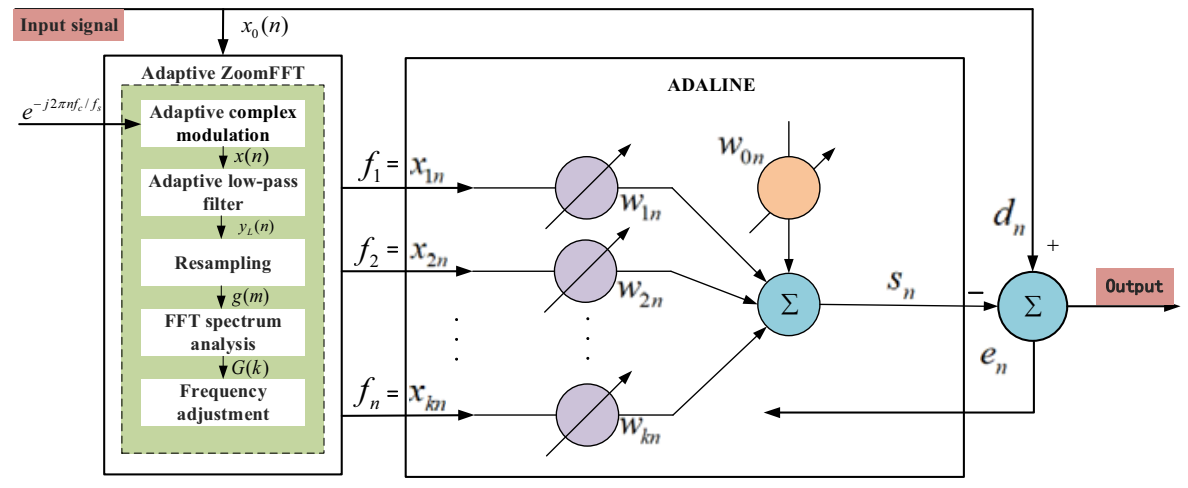

Fig. 3 The principle of Z-ADALINE

The output of adaptive Zoom FFT, frequency components $f=\left[f_{1}, f_{2}, \ldots f_{n}\right]$, is used as the input of ADALINE to calculate $\hat{y}(n)$ by formula $(10) \sim(15)$. Using $x_{0}(n)$ as the expected output, the gradient descent algorithm is used to solve for the minimum point of the error function. At this time, we have defined the cost function. When the cost function Sum of Squared Errors $J(w)=\frac{1}{2} \sum_{i}\left(y(i)-x_{0}(i)\right)^{2}$ gets the minimum value, we obtain the final output result. The advantage of this cost function is that it can be differentiated, which is a convex function. The weight value with the smallest mean square error can be found by the algorithm of gradient descent. In the process of ADALINE training, the process of finding the minimum mean square error is just like going down the mountain. Each algorithm cycle is equivalent to a step down, and the magnitude of the step down depends on the learning rate $\eta$. When the error approaches the minimum, 
ADALINE outputs $a_{m}$ and $b_{m}$, and calculates the final estimation results.

In general, the Z-ADALINE first estimates the frequencies of the harmonics and inter-harmonics by the adaptive Zoom FFT. Then they are inputted into ADALINE. After the iteration convergence of the weight, amplitudes and phases are estimated accurately. This combined algorithm significantly improves the adaptive ability and estimation accuracy of wide-frequency signal.

\section{Simulation results and analysis}

Assuming the simulation signal is:

$$
x(n)=\sum_{m=1}^{M} A_{m} \sin \left(2 \pi f_{m} n+\varphi_{m}\right)+v(n)
$$

Where $M$ is the number signal components, $v(n)$ is the Gaussian white noise and $S N R=30 d B$. In this paper, the wide-frequency signal range is premised from 0 to $2.5 \mathrm{kHz}$. According to the hardware, the sampling frequency is set as $12.8 \mathrm{kHz}$. The high frequency component is above $100 \mathrm{~Hz}$. The power frequency component is about $50 \mathrm{~Hz}$. The low frequency component is below $45 \mathrm{~Hz}$. The simulation signal parameters are shown in Tab 1.

Table 1. The simulation signal parameters

\begin{tabular}{ccccc}
\hline No. & Signal & $\mathbf{A} / \mathbf{V}$ & $f$ & $\varphi \times 180^{\circ} / \pi$ \\
\hline \multirow{3}{*}{1} & Low frequency & 50 & 1.3 & -45 \\
& component & 40 & 1.6 & -10 \\
& & 40 & 2 & -30 \\
\hline 2 & & 50 & 50.3 & -45 \\
\hline
\end{tabular}

\begin{tabular}{ccccc}
\hline & Power frequency & 40 & 50.6 & -10 \\
& component & 20 & 50.9 & 30 \\
\hline \multirow{3}{*}{3} & High frequency & 50 & 2050.3 & -45 \\
& component & 40 & 2050.6 & -10 \\
& 20 & 2050.9 & 30 \\
\hline \multirow{3}{*}{4} & Inter-harmonics & 50 & 114.5 & -45 \\
& & 20 & 115.0 & -10 \\
\hline \multirow{2}{*}{5} & Power & 220 & 50 & 30 \\
\hline
\end{tabular}

In the ADALINE neural network, the learning rates is set to $\eta=6 \times 10^{-5}$ for the low-frequency component and $\eta=3 \times 10^{-6}$ for others. The error threshold is $e=0.01$.

Fig. 4 shows the results of FFT and Zoom FFT in high, middle, and low frequency band. The blue line shows the original output of FFT and the red line shows the output of Zoom FFT. Among the low frequency components, we choose three frequency signals with $1.3 \mathrm{~Hz}, 1.6 \mathrm{~Hz}$, and $2 \mathrm{~Hz}$ respectively. Although the signal result is very weak, we obtain every frequency of them. Among the power frequency component, we also choose three frequencies, separated by $0.3 \mathrm{~Hz}$ range from 50 to $50.9 \mathrm{~Hz}$. The algorithm of this paper represented by the red line effectively distinguishes these frequencies. Among high frequency components and inter-harmonics we obtain completely correct measurement results. The magnification $D$ of different bands is clarified. When the measurement frequency range becomes wider and the sampling frequency increases, it is obvious that the spectrum after using adaptive Zoom FFT can distinguish adjacent frequencies and obtain more accurate frequency. 

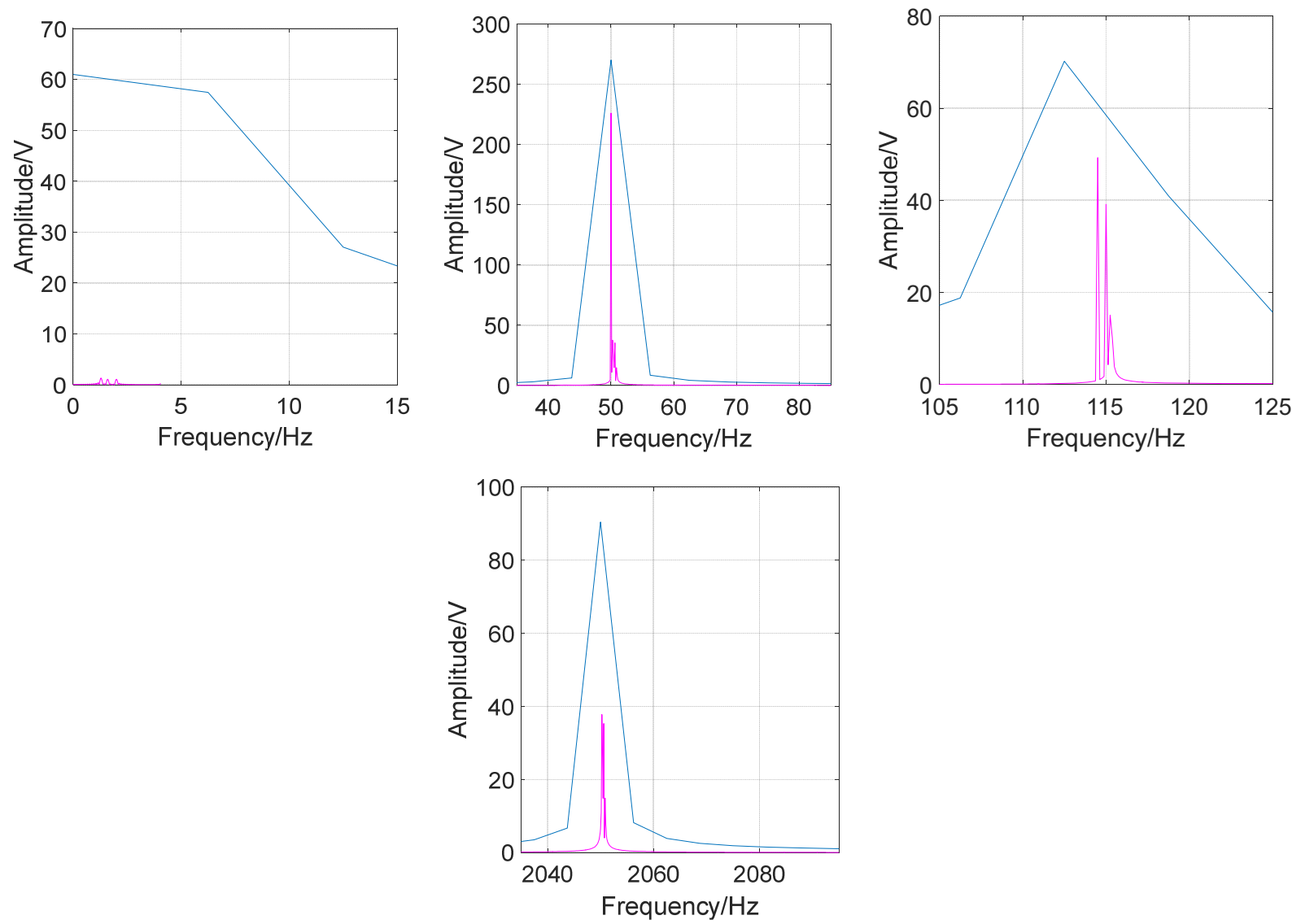

Fig. 4 Simulation results for each frequency component by FFT and Zoom FFT

Obtaining effective frequency components is the premise of realizing the high-precision measurement in this paper. After obtaining accurate frequencies results, we still need to measure amplitude and phase. We combine it with ADALINE and continuously adjust the parameters in the network by the gradient descent algorithm to obtain the results of each frequency band. The detailed results are as follows:

1) Analysis of frequency resolution

Table 2. Comparison of frequency estimation from different algorithms

\begin{tabular}{|c|c|c|c|}
\hline \multirow[b]{2}{*}{$\begin{array}{c}\text { signal } \\
\text { parameters }\end{array}$} & \multicolumn{3}{|c|}{ Analysis of frequency resolution/ $\mathbf{H z}$} \\
\hline & FFT & $\begin{array}{c}\text { Zoom } \\
\text { FFT }\end{array}$ & $\begin{array}{c}\text { Z- } \\
\text { ADALINE } \\
\end{array}$ \\
\hline \multirow{3}{*}{$\begin{array}{l}\text { low frequency } \\
\text { component }\end{array}$} & 0.7 & 1.3 & 1.3 \\
\hline & - & 1.6 & 1.6 \\
\hline & - & 2 & 2 \\
\hline \multirow{3}{*}{$\begin{array}{l}\text { power frequency } \\
\text { component }\end{array}$} & 55.826 & 50.3 & 50.3 \\
\hline & - & 50.6 & 50.6 \\
\hline & - & 50.9 & 50.9 \\
\hline
\end{tabular}

\begin{tabular}{|c|c|c|c|}
\hline \multirow{3}{*}{$\begin{array}{l}\text { high frequency } \\
\text { component }\end{array}$} & 2055 & 2050.3 & 2050.3 \\
\hline & - & 2050.6 & 2050.6 \\
\hline & - & 2050.9 & 2050.9 \\
\hline \multirow{3}{*}{ inter-harmonics } & 114.8 & 114.6 & 114.6 \\
\hline & - & 115.0 & 115.0 \\
\hline & - & 115.3 & 115.3 \\
\hline power frequency & 56.037 & 50 & 50 \\
\hline
\end{tabular}

Tab. 2 shows comparison of frequency estimation from different algorithms. According to the results, the FFT generates spectral leakage and cannot distinguish all frequency components in the wide frequency range. The Zoom FFT can estimate frequencies accurately, and provide them to ADALINE. Z-ADALINE can realize stable frequency resolution of $0.3 \mathrm{~Hz}$ for low, medium, and high frequencies in the frequency band studied in this paper.

2) Analysis of amplitude estimation accuracy

Table 3. Comparison of amplitude estimation from different algorithms

\begin{tabular}{|c|c|c|c|c|c|c|}
\hline \multirow{3}{*}{ Signal parameters } & \multicolumn{6}{|c|}{ Amplitude/ V } \\
\hline & \multicolumn{2}{|c|}{ FFT } & \multicolumn{2}{|c|}{ Zoom FFT } & \multicolumn{2}{|c|}{ Z-ADALINE } \\
\hline & Results & Error\% & Results & Error\% & Results & Error\% \\
\hline \multirow{2}{*}{ Low frequency component } & 61.21 & - & 1.33 & - & 49.48 & 1.04 \\
\hline & - & - & 1.09 & - & 39.53 & 1.19 \\
\hline
\end{tabular}




\begin{tabular}{|c|c|c|c|c|c|c|}
\hline & - & - & 1.09 & - & 39.80 & 0.49 \\
\hline \multirow{3}{*}{ Power frequency component } & 270.13 & - & 37.72 & 24.57 & 50.68 & 1.36 \\
\hline & - & - & 35.37 & 11.59 & 39.87 & 0.31 \\
\hline & - & - & 14.87 & 25.68 & 19.89 & 0.58 \\
\hline \multirow{3}{*}{ High frequency component } & 90.419 & - & 37.76 & 24.49 & 50.35 & 0.70 \\
\hline & - & - & 35.32 & 29.36 & 39.75 & 0.62 \\
\hline & - & - & 14.925 & 25.38 & 20.17 & 0.83 \\
\hline \multirow{3}{*}{ Inter-harmonics } & 70.20 & - & 49.27 & 0.15 & 50.06 & 0.12 \\
\hline & - & - & 39.15 & 2.14 & 39.91 & 0.22 \\
\hline & - & - & 15.06 & 24.69 & 20.14 & 0.70 \\
\hline Power frequency & 222.71 & 0.32 & 219.95 & 0.02 & 219.55 & 0.20 \\
\hline
\end{tabular}

The results in Tab. 3 can be seen that the amplitude and phase estimation results of FFT are inaccurate due to the lack of accurate frequency estimation. Owing to spectral leakage and other problems, the amplitude estimation accuracy of Zoom FFT with higher frequency resolution for the medium and high frequency components is still low. The amplitude estimation errors for the low frequency components are too big for practical application. In contrast, Z-ADALINE has the estimation errors of amplitude estimation which are less than $1 \%$.

3) Analysis of phase estimation accuracy

Table 4. Comparison of phase estimation from different algorithms

\begin{tabular}{|c|c|c|c|c|c|c|}
\hline \multirow{3}{*}{ Signal parameters } & \multicolumn{6}{|c|}{ Phase $\left(\varphi \times 180^{\circ} / \pi\right) / \circ$} \\
\hline & \multicolumn{2}{|c|}{ FFT } & \multicolumn{2}{|c|}{ Zoom FFT } & \multicolumn{2}{|c|}{ Z-ADALINE } \\
\hline & Results & Error\% & Results & Error\% & Results & Error\% \\
\hline \multirow{3}{*}{$\begin{array}{l}\text { Low frequency } \\
\text { component }\end{array}$} & 0.081 & - & -27.36 & 39.20 & -45.25 & 0.55 \\
\hline & - & - & -9.17 & 8.29 & -9.49 & 5.09 \\
\hline & - & - & -42.41 & 41.35 & -29.63 & 1.24 \\
\hline \multirow{3}{*}{ Power frequency component } & 42.372 & - & 31.48 & 21.30 & -45.49 & 1.09 \\
\hline & - & - & -52.54 & - & -10.62 & 6.24 \\
\hline & - & - & 61.68 & - & 30.88 & 2.92 \\
\hline \multirow{3}{*}{$\begin{array}{l}\text { High frequency } \\
\text { component }\end{array}$} & -5.428 & - & 31.56 & - & -45.33 & 0.74 \\
\hline & - & - & -52.48 & - & -10.03 & 0.28 \\
\hline & - & - & 61.77 & - & 30.73 & 2.42 \\
\hline \multirow{3}{*}{ Inter-harmonics } & 44.83 & - & -44.45 & 1.22 & -45.14 & 0.30 \\
\hline & - & - & -6.68 & 33.2 & -10.01 & 0.70 \\
\hline & - & - & 102.1 & 240.03 & 30.19 & 0.63 \\
\hline Power frequency & 60.11 & 0.18 & 59.99 & 0.02 & 60.00 & 0.02 \\
\hline
\end{tabular}

According to the results in Tab. 4 above, which is similar to its amplitude estimation results, the phase estimation results of Zoom FFT are inaccurate. In contrast, the algorithm proposed in this paper has higher estimation accuracy of phase with an error within $0.6^{\circ}$, no matter in which specific frequency band. The data in Tab. 4. shows that Z-ADALINE in this paper has high performance in the phase measurement part.

\section{Conclusion}

We proposed a high-precision estimation algorithm ZADLINE for the wide-frequency signals in power grids. At first, the algorithm uses Zoom FFT to analyze the sampling sequences to obtain accurate estimation results. We have designed the Zoom FFT with adaptive ability, which improved the adaptive abilities of center-frequency, frequency bandwidth and magnification effectively. Secondly, it's results were used as input of ADALINE neural network to estimate amplitudes and phases and realizes complementary advantages. In order to verify the effectiveness of the algorithm in this paper, we design multiple sets of simulations in different frequency bands and conducted detailed analysis. The data relates to low, medium, and high frequency and inter-harmonics in the wide frequency range from 0 to $2.5 \mathrm{kHz}$. The simulation results show that the algorithm can realize the high accurate estimation of signal parameters. $0.3 \mathrm{~Hz}$ resolution frequency can be accurately measured, and the amplitude and phase errors are less than $2.5 \%$. Among them, the amplitude error is within $1 \mathrm{~V}$, and the phase error is less than $0.6^{\circ}$. At the same time, the simulation is strictly based on the wide-frequency signal sampling frequency, which 
has high application value and makes the algorithm easy to applicate in practical projects.

\section{Acknowledgments}

This work is supported by the open foundation of State Key Laboratory of Smart Grid Protection and Control (Grant No. NARI-T-2-2019189)

\section{References}

1. Zainuri M, Atiqi MA, Radzi M, et al. Photovoltaic Integrated Shunt Active PowerFilter with Simpler Adaline Algorithm for Current Harmonic Extraction [J]. Energies, 2018, 11(5): 1152.

2. Du W, Yang H, Ma X. Harmonic / Interharmonic Spectrum Separation Algorithm Based on Fast Independent Component Analysis [J/OL]. Automation of Electric Power Systems:1-10

3. Puhan PS, Sandeep S, Sutar D. Analysis of Adaptive Linear Neural Network (adaline) in Power System Harmonics Signal [J]. Journal on Electrical Engineering, 2018, 11(4)

4. Yu Y, Zhao W, Chen L, et al. Power Measurement Accuracy Analysis in the Presence of Interharmonics [J]. Measurement, 2020: 107484.

5. Jin Zongshuai. High-precision Estimation of Wideband Synchrophasor of Electrical Signals with Heavy Noise [D]. Shangdong University, 2019.

6. Ma Ningning, Xie X, He J, et al. A Review of Research on Broadband Oscillation of High Proportion New Energy and Power Electronic Equipment Power System [J]. Proceedings of The Chinese Society for Electrical, 2020: 1-13.

7. Chen Fan, Yao J, Chang N, et al. Discussion about wide-frequency measurement technology for power electronic power grid $[\mathrm{J}]$. Automation of Electric Power System, 2019, 43(16): 1-10, 57.

8. Fan C, Yao J, Chang N, et al. Discussion on Widefrequency Measurement Technology for Power Electronized Power Grid[J]. Automation of Electric Power Systems ,2019,43(16):1-10+57.

9. Jin Zongshuai, Zhang H, Shi F, et al. Decomposition scheme for wide band signals containing deterministic components and stochastic noise components [J]. Automation of Electric Power Systems, 2019, 43(4): 70-81.

10. Chang G, Chen C, Liu Y, et al. Measuring Power System Harmonics and Interharmonics By an Improved Fast Fourier Transform-based Algorithm [J]. Iet Generation, Transmission \& Distribution, 2008, 2(2): 192-201.

11. Li J, Chen L. Improved Interpolation Algorithm Applied to Power Harmonic Analysis[J]. Automation of Electric Power Systems,2019,43(08):138-146.

12. Gao G, Liang Q, Liu N. A High-precision Frequency Estimation Method Based on Harmonic Expansion Techniqu e[C], 17th International Conference on Optical Communications and Networks (icocn2018), International Society for Optics and Photonics, 2019: 110482.

13. Li L. Research on Related Technology of Harmonic Control of Power System [D]. Zhejiang University, 2011.

14. Cai Z. Research on Adaptive Measurement Technology of Electric Parameters [D]. Zhejiang University, 2009.

15. Goh ZP, Radzi MAM, Von Thien Y, et al. Hybrid Fftadaline Algorithm with Fast Estimation of Harmonics in Power System [J]. Iet Signal Processing, 2016, 10(8): 855-864.

16. Chen G, Cai Z, Chen L. Interharmonic Parameter Estimation Based on Root-music and Adaline Neural Network [J]. Journal of Zhejiang University (engineering Science), 2011, 45(1): 173-177.

17. Agrawal S, Mohanty SR, Agarwal V. Harmonics and Inter Harmonics Estimation of Dfig Based Standalone Wind Power System By Parametric Techniques [J]. International Journal of Electrical Power \& Energy Systems,2015, 67: 52-65.

18. Yu S, Zhang L, Xie W, et al. Fast and High-precision Spectrum Analysis of Optical Frequency Domain Reflectometer Based on Genetic Algorithm and Zoom FFT [J]. Acta Optica Sinica, 2017, 37(4): 406003.

19. Dao X, Gao M, Ke Z. Research on Improved Algorithm of Frequency Estimation Based on Complex Modulation [J]. Engineering Letters, 2018, 26(4).

20. Goswami S, Sarkar A, Sengupta S. Advancements in Instrumentation and Control in Applied System Applications[M]. Igi Global, 2020: 68-97.

21. Garanayak P, Naayagi R, Panda G. A High-speed Master-slave Adaline for Accurate Power System Harmonic and Inter-harmonic Estimation [J]. Ieee Access, 2020, 8: 51918-51932.

22. Sujith M, Padma S. Optimization of Harmonics with Active Power Filter Based on Adaline Neural Network[J]. Microprocessors and Microsystems, 2020, 73: 102976.

23. Nanda S, Dash PK. A Gauss-newton Adaline for Dynamic Phasor Estimation of Power Signals and Its Fpga Implementation [J]. Ieee Transactions on Instrumentation and Measurement, 2016, 67(1): 4556.

24. Wang W. Research on Improved Adaptive ZoomFFT Algorithm [J]. Electronic Technology Application, 2006, (7): 83-85. 